\title{
Symmetry Types and Phase-Shift Synchrony in Networks
}

\author{
Martin Golubitsky \\ Leopold Matamba Messi \\ Lucy E. Spardy \\ Mathematical Biosciences Institute \\ The Ohio State University \\ Columbus, OH 43210
}

May 6, 2015

\begin{abstract}
In this paper we discuss what is known about the classification of symmetry groups and patterns of phase-shift synchrony for periodic solutions of coupled cell networks. Specifically, we compare the lists of spatial and spatiotemporal symmetries of periodic solutions of admissible vector fields to those of equivariant vector fields in the three cases of $\mathbb{R}^{n}$ (coupled equations), $\mathbb{T}^{n}$ (coupled oscillators), and $\left(\mathbb{R}^{k}\right)^{n}$ where $k \geq 2$ (coupled systems). To do this we use the $H / K$ Theorem of Buono and Golubitsky applied to coupled equations and coupled systems and prove the $H / K$ theorem in the case of coupled oscillators. Josić and Török [17] prove that the $H / K$ lists for equivariant vector fields and admissible vector fields are the same for transitive coupled systems. We show that the corresponding theorem is false for coupled equations. We also prove that the pairs of subgroups $H \supset K$ for coupled equations are contained in the pairs for coupled oscillators which are contained in the pairs for coupled systems. Finally, we prove that patterns of rigid phase-shift synchrony for coupled equations are contained in those of coupled oscillators and those of coupled systems.
\end{abstract}

Keywords: phase-shift synchony, periodic solutions, coupled cell networks, symmetry AMS Subject Classification: 34C25, 34C14

\section{Introduction}

Many biological phenomena, such as respiration [1, 2], locomotion [4, 6, 12, 13, 25], or rivalry [5] are characterized by robust rhythmic patterns that exhibit particular phase relationships or phaseshifts. The neuronal networks responsible for these behaviors can be represented as coupled systems of differential equations that exhibit periodic behavior corresponding to these phase-shift patterns. These phase relationships appear to occur robustly in nature; hence, it is reasonable to utilize models in which the phase-shifts are rigid, that is, they are preserved under small perturbations of the corresponding network of differential equations.

It is well known that rigid phase-shifts in networks of differential equations may be caused by the symmetries of the underlying network. Stewart et al. $[19,16,11]$ developed a framework for studying coupled systems of differential equations that associates to each directed graph a collection of admissible vector fields. These authors and others have then studied properties of solutions and of bifurcations in admissible systems. 
The fact that rigid phase-shifts are also informed by the state spaces of the network nodes is frequently overlooked. Classically, modelers often use state spaces for individual nodes that are either one-dimensional $\mathbb{R}$ (smoothed out integrate and fire systems), circles $\mathbb{T}$ (oscillators), or multidimensional $\mathbb{R}^{k}$ where $k \geq 2$ (Hodgkin-Huxley neurons). We call these cases: coupled equations, coupled oscillators, and coupled systems. This paper studies the similarities and the differences between patterns of phase-shift synchrony forced by network symmetry in these three contexts, both for the class of admissible vector fields and for the less restrictive class of equivariant vector fields.

\section{Examples of rigid phase-shifts for periodic solutions}

Before presenting our results we recall some terminology. Specifically, we discuss rigid phase-shifts, patterns of phase-shift synchrony, and their relationship to symmetry. Suppose that a network has $n$ nodes and state variables $\left(x_{1}, \ldots, x_{n}\right)$.

Definition 1.1. A $T$-periodic solution $X(t)=\left(x_{1}(t), \ldots, x_{n}(t)\right)$ of an admissible system has a phase-shift $\theta_{i j}$ if there are two nodes $i$ and $j$ such that

$$
x_{j}(t)=x_{i}\left(t+\theta_{i j} T\right) .
$$

This phase-shift is rigid if a perturbed admissible system has a perturbed $\tilde{T}$-periodic solution $\tilde{X}(t)=\left(\tilde{x}_{1}(t), \ldots, \tilde{x}_{n}(t)\right)$ such that

$$
\tilde{x}_{j}(t)=\tilde{x}_{i}\left(t+\theta_{i j} \tilde{T}\right)
$$

with the same phase-shift.

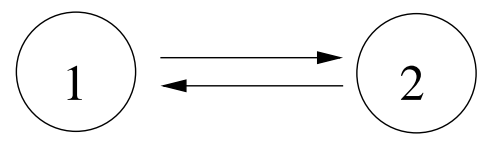

(a) Two identical node, identical coupling network with $\sigma=\left(\begin{array}{ll}1 & 2\end{array}\right)$ symmetry.

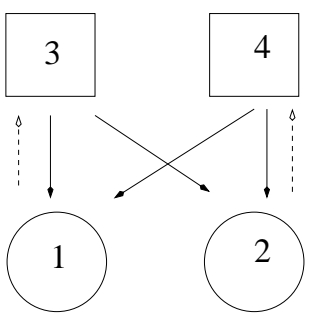

(b) Four-cell network with $\sigma=\left(\begin{array}{ll}1 & 2\end{array}\right)\left(\begin{array}{ll}3 & 4\end{array}\right)$ symmetry.

Figure 1: Examples of networks with $\mathbf{Z}_{2}$ symmetry.

To illustrate different rigid phase-shifts, consider the networks shown in Figure 1. The admissible systems corresponding to Figure 1(a) have the form

$$
\begin{aligned}
& \dot{x}_{1}=f\left(x_{1}, x_{2}\right) \\
& \dot{x}_{2}=f\left(x_{2}, x_{1}\right)
\end{aligned}
$$

and the admissible systems corresponding to Figure 1(b) have the form

$$
\begin{aligned}
& \dot{x}_{1}=h\left(x_{1}, \overline{x_{3}, x_{4}}\right) \\
& \dot{x}_{2}=h\left(x_{2}, \overline{x_{3}, x_{4}}\right) \\
& \dot{x}_{3}=g\left(x_{3}, x_{1}\right) \\
& \dot{x}_{4}=g\left(x_{4}, x_{2}\right)
\end{aligned}
$$


where $x_{1}, x_{2} \in P ; x_{3}, x_{4} \in Q$ and $P$ and $Q$ are phase spaces of individual nodes. The overline indicates that $h(a, b, c)=h(a, c, b)$.

As is well known, there are stable anti-phase periodic solutions of coupled systems and coupled oscillators for (1.1) having the form

$$
x_{2}(t)=x_{1}\left(t+\frac{1}{2} T\right)
$$

(found by Hopf bifurcation), but it is less often discussed that such solutions cannot exist for coupled equations. Similarly, there are stable periodic solutions of coupled systems and of coupled oscillators for (1.2) having the form

$$
x_{2}(t)=x_{1}\left(t+\frac{1}{2} T\right) \quad x_{4}(t)=x_{3}\left(t+\frac{1}{2} T\right),
$$

but this solution does not exist for coupled equations. Each of these solution types are generated by the $\sigma$ symmetry of their associated networks (see Figure 1).

The second network illustrates the subtle fact that a pattern of phase-shift synchrony can be forced by a symmetry on a quotient network, rather than by a symmetry on the network itself. See $[15,10,14]$. Specifically, (1.2) can also have a periodic solution of the form

$$
x_{2}(t)=x_{1}(t) \quad x_{4}(t)=x_{3}\left(t+\frac{1}{2} T\right)
$$

that is not generated by a network symmetry of Figure $1(\mathrm{~b})$. Note that $\Delta=\left\{x_{1}=x_{2}\right\}$ is a flowinvariant subspace for every admissible vector field in (1.2) and the equations for the admissible vector fields restricted to $\Delta$ have the form

$$
\begin{aligned}
& \dot{x}_{1}=h\left(x_{1}, \overline{x_{3}, x_{4}}\right) \\
& \dot{x}_{3}=g\left(x_{3}, x_{1}\right) \\
& \dot{x}_{4}=g\left(x_{4}, x_{1}\right) .
\end{aligned}
$$

These equations correspond to the quotient network given in Figure 2 and this quotient network has a symmetry $\tau=(34)$. It is the symmetry $\tau$ on the quotient network that generates the solution type (1.3). Specifically, stable solutions where $x_{3}(t)$ and $x_{4}(t)$ are in anti-phase can be found by Hopf bifurcation for coupled systems and numerically for coupled oscillators. In these solutions $x_{1}(t)$ oscillates at twice the frequency of $x_{3}(t)$. It follows that in Network $1(\mathrm{~b}), x_{2}(t)=x_{1}\left(t+\frac{1}{2} T\right)=x_{1}(t)$.

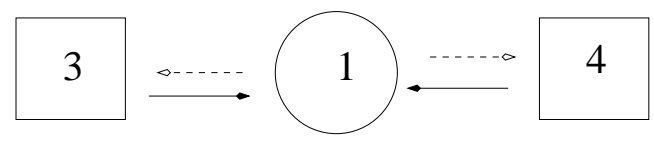

Figure 2: $\mathbf{Z}_{2}$-symmetric, three-cell quotient network of the network in Figure 1(b).

\section{Patterns of phase-shift synchrony}

In a series of papers, Stewart and Parker [20, 21] and Golubitsky, Romano, and Wang [8, 9] proved that in path connected networks of either coupled equations or coupled systems, rigid phase-shifts always result from symmetry. However, that symmetry may be a symmetry of a quotient network, rather than a network symmetry.

More precisely, we define: 
Definition 1.2. A pattern of phase-shift synchrony is a subset of pairs of nodes $i$ and $j$ and phaseshifts $0 \leq \theta_{i j}<1$. A $T$-periodic solution $x(t)=\left(x_{1}(t), \ldots, x_{n}(t)\right)$ exhibits this pattern of synchrony if

$$
x_{j}(t)=x_{i}\left(t+\theta_{i j} T\right)
$$

for all designated pairs $i, j$ and the $\theta_{i j}$ are rigid.

These four papers [20, 21, 8, 9] prove the following: Suppose that a periodic solution $X(t)$ exhibits a pattern of phase-shift synchrony. Then the polydiagonal defined by

$$
\triangle=\left\{X=\left(x_{1}, \ldots, x_{n}\right): x_{i}=x_{j} \text { when } \theta_{i j}=0\right\}
$$

is flow-invariant. Moreover, there is a cyclic symmetry $\tau$ on the quotient network corresponding to $\triangle$ that generates all of the nonzero $\theta_{i j}$ in the pattern of phase-shift synchrony.

\section{Symmetry groups of periodic solutions for equivariant systems}

A symmetry of a system of differential equations

$$
\dot{X}=F(X)
$$

is a linear map $\sigma$ that maps solutions to solutions. It is well known that $\sigma$ is a symmetry if and only if the equivariance condition

$$
F(\sigma X)=\sigma F(X)
$$

holds.

We now recall how phase-shifts can be defined by symmetries. Suppose that (1.5) is a general system of differential equations with symmetry group $\Gamma$; that is, $F(\gamma X)=\gamma F(X)$ for all $\gamma \in \Gamma$. We define the spatial symmetries and the spatiotemporal symmetries of a $T$-periodic solution $X(t)$ to be

$$
\begin{array}{ll}
K=\{\gamma \in \Gamma: \gamma X(t)=X(t) \forall t\} & \text { spatial symmetries } \\
H=\{\gamma \in \Gamma: \gamma\{X(t)\}=\{X(t)\}\} & \text { spatiotemporal symmetries }
\end{array}
$$

If $\gamma \in H$, then by definition $\gamma X(0)=X(\theta T)$ for some $0 \leq \theta<1$. Uniqueness of solutions with a given initial condition implies that

$$
\gamma X(t)=X(t+\theta T)
$$

and $\gamma, \theta$ together form a spatiotemporal symmetry of the periodic solution. Moreover, if $\gamma$ is an order two element, then either $\theta=0$ or $\theta=\frac{1}{2}$. It is straightforward to show that $H / K$ is cyclic. Finally, we recall that $H, K$, and $\theta$ are rigid [10] in the sense that small equivariant perturbations lead to periodic solutions with the same $H, K$, and $\theta$.

Note that

$$
\operatorname{Fix}(K)=\{x: \sigma x=x \quad \forall \sigma \in K\}
$$

is flow-invariant and corresponds to the flow-invariant subspace $\triangle$ when discussing phase-shifts. Specifically, $K$ identifies synchronous nodes and $H / K$ is a subgroup of symmetries on the quotient network corresponding to $\operatorname{Fix}(K)[16,11]$. 


\section{Synchrony, phase-shifts, and multirhythms in networks}

Consider an $n$-node network with symmetry group $\Gamma$ and an admissible system with a $T$-periodic solution $X(t)=\left(x_{1}(t), \ldots, x_{n}(t)\right)$. We can relate symmetries of $X(t)$ to synchrony and phase-shift synchrony as follows. We write a symmetry $\sigma$ as a unique product of disjoint cycles; indeed, after renumbering, we suppose that a factor of $\sigma$ is the $\ell$-cycle $(12 \cdots \ell)$.

First, assume that $\sigma$ is in $K \subset \Gamma$. Then $\sigma X(t)=X(t)$ implies the synchrony condition

$$
x_{1}(t)=\cdots=x_{\ell}(t) .
$$

Second, assume that $K=\mathbf{1}$ and $\sigma$ is a generator of the cyclic group $H / K=H$. Then, the symmetry condition $\sigma\{X(t)\}=\{X(t)\}$ implies the phase-shift synchrony conditions

$$
x_{j}(t)=x_{1}\left(t+\frac{j-1}{\ell} T\right)
$$

for $j=1, \ldots, \ell$; that is, the solution is a discrete rotating wave on the first $\ell$ nodes. In addition to the rotating waves corresponding to each cyclic factor of $\sigma$, there are relationships between the phases on different cyclic factors, which generates multirhythms. For example, if $\sigma=\left(\begin{array}{lll}1 & 2 & 3\end{array}\right)(45)(6)$, then the $T$-periodic solution $X(t)$ satisfies

$$
\begin{aligned}
& x_{1}(t)=x_{1}\left(t+\frac{1}{2} T\right) \\
& x_{2}(t)=x_{1}\left(t+\frac{1}{3} T\right) \\
& x_{3}(t)=x_{1}\left(t+\frac{2}{3} T\right) \\
& x_{4}(t)=x_{4}\left(t+\frac{1}{3} T\right) \\
& x_{5}(t)=x_{4}\left(t+\frac{1}{2} T\right) \\
& x_{6}(t)=x_{6}\left(t+\frac{1}{6} T\right) .
\end{aligned}
$$

So the nodes divide into three sets. The first three nodes have a discrete rotating wave with onethird period phase-shifts and oscillate with period $T / 2$. The fourth and fifth nodes are in antiphase and oscillate with period $T / 3$. The sixth node oscillates with period $T / 6$.

\section{The principal questions}

Networks of differential equations have phase spaces that are products of the phase spaces for the individual nodes. We have emphasized this by the terms coupled equations, coupled oscillators, and coupled systems. Symmetry groups $\Gamma$ of networks consist of permutations that preserve network architecture. It follows that every admissible vector field on a network is $\Gamma$-equivariant, but it is not the case that every $\Gamma$-equivariant vector field is admissible. See Section 4 . In this paper, we explore properties of periodic solutions that are rigid for equivariant systems $(H \supset K$ pairs $)$ and properties that are rigid for admissible systems (synchrony and phase-shift synchrony). As we have seen these concepts are related. Indeed, for equivariants we distinguish the three network cases by the terms equivariant equations, equivariant oscillators, and equivariant systems, and similarly for admissibles. Given a coupled cell network, we ask:

(a) For which patterns of phase-shift synchrony do there exist periodic solutions of admissible vector fields exhibiting that particular pattern?

(b) For which network symmetry subgroups $H \supset K$ do there exist periodic solutions of admissible vector fields having those particular symmetries? 
Note that question (b) is a special case of question (a) and deals only with those patterns of phase-shift synchrony that are attributable to network symmetry — not those that are related to symmetries on proper quotient networks. Our current state of knowledge is summarized by the containments shown in Figure 3. For a fixed network, each rectangular box refers to the set of $H \supset K$ pairs for which there exists a vector field having a (hyperbolic) periodic solution with space symmetries $K$ and spatiotemporal symmetries $H$. The bottom row refers to the list with admissible vector fields, whereas the top row refers to the much larger class of equivariant vector fields. The three columns refer to phase spaces in the cases of coupled equations, coupled oscillators, and coupled systems.

$$
\begin{array}{cc}
\text { Theorem 3.7(a) } & \text { Theorem 3.7(b) } \\
\text { Example 3.8(a) } & \text { Example 3.8(b) }
\end{array}
$$

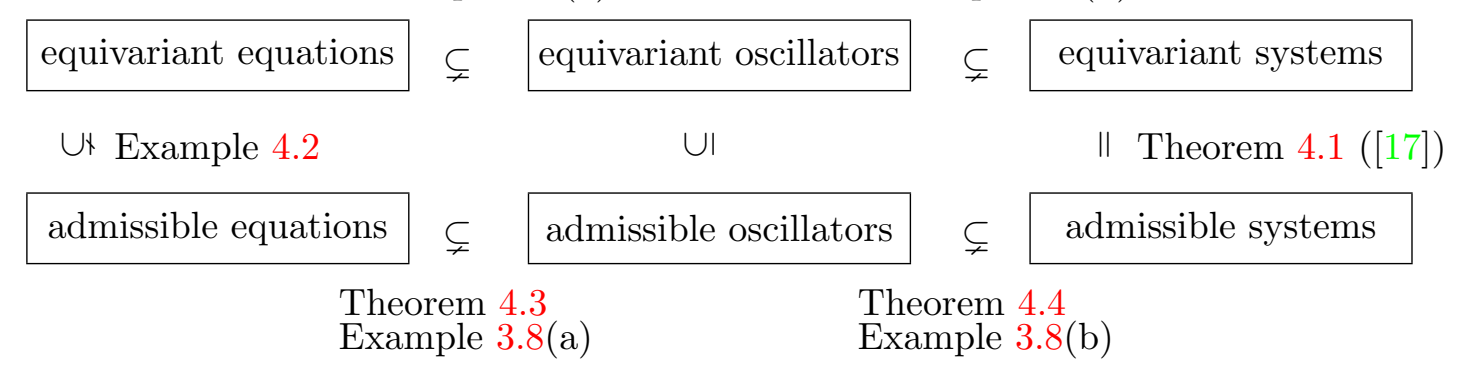

Figure 3: Comparison of $H \supset K$ symmetry pairs of periodic solutions of admissible and equivariant vector fields for equations, oscillators, and systems in transitive coupled-cell networks.

Begin by observing that an $H / K$ pair that occurs for an admissible vector field also occurs for an equivariant vector field because every admissible vector field is equivariant. In general, we do not know that there exists an admissible vector field with a $H \supset K$ periodic solution even when such a periodic solution exists for an equivariant vector field. This fact was proved by Josić and Török [17] in the case of coupled systems on transitive networks. We show by example (see Example 4.2) that the corresponding result is false for coupled equations. Finally, this statement is still unresolved for coupled oscillators. These comments are summarized by the vertical containments between the two rows in Figure 3.

Buono and Golubitsky [3] answered a question analagous to (b) by determining the possible subgroup pairs $H \supset K$ for equivariant vector fields of a finite group acting on $\mathbb{R}^{N}$. Their result is the $H / K$ Theorem which gives necessary and sufficient conditions for the existence of a periodic solution to some equivariant system having symmetries $H \supset K$. One of our main results is the $H / K$ Theorem for permutation actions of $\Gamma$ on $\mathbb{T}^{n}$ (see Theorem 3.4), which is the case of coupled oscillators. This theorem enables us to prove that the containments on the top row of Figure 3 are valid. See Theorem 3.7. We also prove by different arguments that the containments of admissible pairs $H \supset K$ for coupled equations, coupled oscillators, and coupled systems given in the second row of Figure 3 are valid. See Theorems 4.3 and 4.4. Finally, not every $H / K$ pair for coupled systems is one for coupled oscillators and not every pair for coupled oscillators is one for coupled equations. See Example 3.8.

Most of our results correspond to question (b); however, we do comment on question (a). Specificallly, we prove Theorem 5.1 that shows that patterns of phase-shift synchrony for coupled equations are patterns for coupled oscillators and coupled systems. We do not yet know whether patterns of phase-shift synchrony for coupled oscillators are always patterns of phase-shift synchrony for coupled systems, though this seems likely. See Remark 5.2. 


\section{The structure of the paper}

In Section 2 we define networks and network admissible vector fields. Section 3 considers periodic solutions of equivariant vector fields in the context where the group $\Gamma$ is a permutation group, specifically the symmetry group of a network. In this section we prove the $H / K$ Theorem (Theorem 3.4) for equivariant vector fields when the phase space is $T^{N}$, the phase space for coupled oscillators. The cases of coupled equations and coupled systems follow from the $H / K$ Theorem of Buono and Golubitsky [3] (see Theorem 3.1 specialized to permutation actions of symmetry groups in Corollaries 3.2 and 3.3). In Section 4 we discuss which of the possible symmetry pairs $H \supset K$ actually have periodic solutions associated to some admissible vector field, and we do so in the three contexts of equations, oscillators, and systems. Previously, Josić and Török [17] proved that every possible symmetry pair occurs in the coupled systems case of path-connected (or transitive) networks. Example 4.2 shows that surprisingly the same statement is not valid for coupled equations. As noted, Section 5 comments on question (a).

\section{Admissible vector fields and patterns of phase-shift synchrony}

Golubitsky, Stewart and coauthors [19, 16, 11] laid the foundations for a theory of coupled systems. In these papers, they formalized the language of coupled-cell networks and established some of the generic properties of steady-state and periodic solutions in the class of admissible systems.

In this theory a network is defined by a set of $n$ nodes and a set of directed arrows between nodes. Each arrow $a$ has a head node $\mathcal{H}(a)$ and a tail node $\mathcal{T}(a)$. The input set $I(i)=\left\{a_{1}, \ldots, a_{p}\right\}$ consists of all arrows whose head node is $i$. Admissible systems corresponding to the network are obtained by first choosing a phase space $P_{j}$ for each node $j$ and associating to each input set $I(i)$ a vector of phase variables $x_{I(i)}$ as follows. We define $x_{I(i)}=\left(x_{\mathcal{T}\left(a_{1}\right)}, \ldots, x_{\mathcal{T}\left(a_{p}\right)}\right) \in P_{\mathcal{T}\left(a_{1}\right)} \times \cdots \times P_{\mathcal{T}\left(a_{p}\right)}$. Each admissible system of an $n$-node network has the form

$$
\dot{x}_{i}=f_{i}\left(x_{i}, x_{I(i)}\right)
$$

The general theory allows for nodes to be cell-equivalent (they have the same phase space), arrows to be edge-equivalent (the coupling formulas are identical), and nodes to be input equivalent (the functions $f_{i}$ at two nodes are identical).

We follow [16] and define a network architecture to be a directed graph with annotated nodes and annotated arrows. We assume:

(a) If two nodes $i$ and $j$ are cell equivalent, then $P_{i}=P_{j}$.

(b) If two arrows are edge equivalent, their head nodes (resp. tail nodes) have the same node type.

(c) If two arrows $j \rightarrow i$ and $k \rightarrow i$ have the same arrow type, then $f_{i}$ is invariant under swapping $x_{j}$ and $x_{k}$; that is, the couplings indicated by these pair of arrows are identical.

(d) Two nodes $i$ and $j$ are input equivalent if there is an edge type preserving bijection between $I(i)$ and $I(j)$. If nodes $i$ and $j$ are input equivalent, then the functions $f_{i}$ and $f_{j}$ are identical.

\section{Network symmetries and synchrony subspaces}

A symmetry $\sigma$ of a network is a permutation of its nodes such that for every pair of nodes $i$ and $j$ there is an arrow type preserving bijection between the set of arrows connecting node $i$ to node $j$ and the set of arrows connecting node $\sigma(i)$ to node $\sigma(j)$. It follows that nodes $i$ and $\sigma(i)$ are input equivalent. Each symmetry $\sigma$ acts as a permutation on the phase space $P=P_{1} \times \cdots \times P_{n}$. 
A synchrony subspace is a polydiagonal that is flow-invariant for all admissible vector fields. It is well known that fixed-point subspaces are flow-invariant for equivariant vector fields and hence for admissible vector fields. It was shown in $[19,16]$ that there are synchrony subspaces that are not fixed-point subspaces. This observation was both surprising and important in the development of coupled cell theory. See $[16,11]$.

Synchrony subspaces can be determined from the network architecture by a combinatorial condition. A coloring is balanced if for every pair of nodes $i$ and $j$ having the same color, there is an edge type preserving bijection between $I(i)$ and $I(j)$ that also preserves tail cell colors. It follows that nodes with the same color in a balanced coloring are input equivalent. So we can associate a polydiagonal $\triangle$ to each balanced coloring as follows. Let

$$
\triangle=\left\{X=\left(x_{1}, \ldots, x_{n}\right): x_{i}=x_{j} \text { whenever the colors of } i \text { and } j \text { are the same }\right\}
$$

This polydiagonal makes sense since $P_{i}=P_{j}$ whenever nodes $i$ and $j$ are input equivalent. Theorem 4.3 in [16] proves that there is a 1:1 correspondence between balanced colorings and synchrony subspaces.

\section{Equivariant $H / K$ theorems for permutation actions}

In this section we consider permutation actions that arise naturally from symmetry groups of coupled-cell networks and address question (b); specifically we answer the following questions:

Which pairs of subgroups $K \subseteq H$ of $\Gamma$ are spatial and spatiotemporal symmetries of a hyperbolic periodic solution of some equivariant vector field when the phase space is $\mathbb{R}^{n}$ (coupled equations), $\mathbb{T}^{n}$ (coupled oscillators), $\mathbb{R}^{k_{1}} \times \cdots \times \mathbb{R}^{k_{n}}$ for $k_{j} \geq 2$ (coupled systems)?

The $H / K$ Theorem of Buono and Golubitsky $[3,10]$ answers this question in the general case of orthogonal actions of a finite group $\Gamma$ on $\mathbb{R}^{N}$ (see Theorem 3.1) and hence leads to a specification of spatiotemporal symmetries in the cases of equivariant coupled equations (Corollary 3.3) and equivariant coupled systems (Corollary 3.2). The principal new result in this section is Theorem 3.4 - the equivariant $H / K$ Theorem for coupled oscillators.

Before stating the $H / K$ Theorem, we recall some terminology and notation. Let $N_{\Gamma}(K)$ denote the normalizer of $K$ in $\Gamma$, let $\Gamma_{x}$ denote the isotropy subgroup of a point $x \in \mathbb{R}^{N}$, and define the variety

$$
L_{K}:=\bigcup_{\gamma \notin K} \operatorname{Fix}(\gamma) \cap \operatorname{Fix}(K)=\left\{x \in \operatorname{Fix}(K): K \subsetneq \Gamma_{x}\right\} .
$$

Theorem 3.1 (H/K Theorem [3, 10]). Let $\Gamma$ be a finite group acting orthogonally on $\mathbb{R}^{N}$ and let $H \supseteq K$ be subgroups of $\Gamma$. There exists a hyperbolic periodic solution for a $\Gamma$-equivariant vector field on $\mathbb{R}^{N}$ with spatial symmetries $K$ and spatiotemporal symmetries $H$ (see (1.6)) if and only if

(a) $K$ is a normal subgroup of $H$ and $H / K$ is cyclic.

(b) $K$ is an isotropy subgroup.

(c) $\operatorname{dim} \operatorname{Fix}(K) \geq 2$ and if $\operatorname{dim} \operatorname{Fix}(K)=2$, then either $H=K$ or $H=N_{\Gamma}(K)$.

(d) $H$ fixes a connected component of $\operatorname{Fix}(K) \backslash L_{K}$. 


\section{$H / K$ theorems for permutation actions on network phase spaces}

The classifications of the symmetries of periodic orbits for equivariant equations and equivariant systems are corollaries of the equivariant $\mathrm{H} / \mathrm{K}$ theorem in the special case of permutation actions.

Corollary $3.2\left(H / K\right.$ Theorem for equivariant systems). Let $\mathbb{R}^{N}=\mathbb{R}^{k_{1}} \times \cdots \times \mathbb{R}^{k_{n}}$ where $k_{j} \geq 2$ and let $\Gamma$ act by permutations on $\mathbb{R}^{N}$. The subgroups $H \supseteq K$ of $\Gamma$ are the spatiotemporal and spatial symmetries of a hyperbolic periodic solution of some $\Gamma$-equivariant vector field on $\mathbb{R}^{N}$ if and only if

(a) $K$ is normal in $H$ and $H / K$ is cyclic.

(b) $K$ is an isotropy subgroup of $\Gamma$.

(c) If $\operatorname{dim} \operatorname{Fix}(K)=2$, then $H=K=\Gamma$.

Proof. Recall that fixed-point subspaces of permutation actions are polydiagonals. Since each factor in the phase space $\mathbb{R}^{N}$ has dimension at least two, it follows that $\operatorname{Fix}(\gamma) \cap \operatorname{Fix}(K)$ has codimension at least two in $\operatorname{Fix}(K)$ when $\gamma \notin K$. Thus, for equivariant systems, Theorem 3.1(d) is satisfied as $\operatorname{Fix}(K) \backslash L_{K}$ is connected. Moreover, Theorem 3.1(c) undergoes two changes. First, the condition on the lower bound of the dimension of $\operatorname{Fix}(K)$ is always satisfied. Second, $\operatorname{dim} \operatorname{Fix}(K)=2$ only if $\mathcal{P}=\mathbb{R}^{2} \times \cdots \times \mathbb{R}^{2}$ and $K$ acts transitively on the $\mathbb{R}^{2}$ factors. If follows that $K=H=\Gamma$ since $K$ is an isotropy subgroup.

Corollary 3.3 ( $H / K$ Theorem for equivariant equations). Suppose that $\Gamma$ acts on $\mathbb{R}^{n}$ by permutations. The subgroups $H \supseteq K$ of $\Gamma$ are the spatiotemporal and spatial symmetries of a hyperbolic periodic solution of some $\Gamma$-equivariant vector field on $\mathbb{R}^{n}$ if and only if

(a) $K$ is normal in $H$ and $H / K$ is cyclic.

(b) $K$ is an isotropy subgroup of $\Gamma$.

(c) $\operatorname{dim} \operatorname{Fix}(K) \geq 2$ and if $\operatorname{dim} \operatorname{Fix}(K)=2$, then $H=K$.

(d) $H$ fixes a connected component of $\operatorname{Fix}(K) \backslash L_{K}$.

Proof. Theorem 3.1 (c) is modified in the case of equivariant equations, whereas conditions (a), (b), and (d) are the same. If the dimension of $\operatorname{Fix}(K)$ is two, then after renumbering of the nodes

$$
\operatorname{Fix}(K)=\left\{x \in \mathbb{R}^{n}: x_{1}=\cdots=x_{\ell}, x_{\ell+1}=\cdots=x_{n}\right\} \quad \text { for some } 1 \leq \ell \leq \frac{n}{2} .
$$

Consequently, either $K=N_{\Gamma}(K)$ (in which case $H=K$ ) or $N_{\Gamma}(K) / K \cong \mathbf{Z}_{2}$ (in which case $H=K$ or $H=N_{\Gamma}(K)$ ). However, if $N_{\Gamma}(K) / K \cong \mathbf{Z}_{2}$, then $L_{K}=\left\{x \in \mathbb{R}^{n}: x_{1}=\cdots=x_{n}\right\}$ and $\operatorname{Fix}(K) \backslash L_{K}$ splits into two connected components that are interchanged by the elements of $N_{\Gamma}(K) \backslash K$. Thus, if $\operatorname{Fix}(K)$ has dimension two, then $H=K$.

\section{Symmetry pairs for equivariant oscillators}

We now state and prove the $H / K$ Theorem for equivariant oscillators.

Theorem 3.4 ( $H / K$ Theorem for equivariant oscillators). Suppose that $\Gamma$ acts on $\mathbb{T}^{n}$ by permutations. The subgroups $H \supseteq K$ of $\Gamma$ are the spatiotemporal and spatial symmetries of a hyperbolic periodic solution of some $\Gamma$-equivariant oscillators if and only if

(a) $K$ is normal in $H$ and $H / K$ is cyclic.

(b) $K$ is an isotropy subgroup. 
(c) If $\operatorname{dim} \operatorname{Fix}(K)=1$, then $K=H=\Gamma$.

If $\operatorname{dim} \operatorname{Fix}(K)=2$, then either $H=K$ or $H=N_{\Gamma}(K)$ and $N_{\Gamma}(K) / K \cong \mathbf{Z}_{2}$

(d) $H$ fixes a connected component of $\operatorname{Fix}(K) \backslash L_{K}$.

As in the $H / K$ Theorem, we show in the proof that the vector field may be chosen so that the periodic orbit is asymptotically stable.

Proof of necessity. The proof that properties (a), (b) and (d) are necessary is identical to that of Buono and Golubitsky [10] for the equivariant $\mathrm{H} / \mathrm{K}$ theorem on $\mathbb{R}^{N}$. The main difference occurs when verifying the necessity of assertion $(c)$.

If $\operatorname{dim} \operatorname{Fix}(K)=1$, then $\operatorname{Fix}(K)=\left\{(\theta, \ldots, \theta) \in \mathbb{T}^{n}: \theta \in \mathbf{S}^{1}\right\}$ and since $K$ is an isotropy subgroup of a point in $\operatorname{Fix}(K), K$ must equal $\Gamma$ and it follows that $H=K=\Gamma$.

If $\operatorname{dim} \operatorname{Fix}(K)=2$, then after renumbering $\operatorname{Fix}(K)$ must have the form (3.2).

$$
\operatorname{Fix}(K)=\left\{\left(\theta_{1}, \ldots, \theta_{n}\right) \in \mathbb{T}^{n}: \theta_{1}=\cdots=\theta_{\ell} \text { and } \theta_{\ell+1}=\cdots=\theta_{n}\right\}
$$

Next, since $K$ is an isotropy subgroup, any element $\gamma \in \Gamma$ that permutes the first $\ell$ coordinates (and hence the last $n-\ell$ coordinates) must be in $K$. Furthermore, since the normalizer $N_{\Gamma}(K)$ acts on $\operatorname{Fix}(K)$ by permutation, the elements of $N_{\Gamma}(K)$ that act nontrivially on $\operatorname{Fix}(K)$ must swap the two blocks. Therefore, either $N_{\Gamma}(K)$ acts trivially on $\operatorname{Fix}(K)$ in which case $N_{\Gamma}(K)=H=K$ or $N_{\Gamma}(K) / K=\mathbf{Z}_{2}$ in which case either $H=K$ or $H=N_{\Gamma}(K)$. So when $\operatorname{dim} \operatorname{Fix}(K)=2$, either $H=K$ or $H=N_{\Gamma}(K)$ with $N_{\Gamma}(K) / K=\mathbf{Z}_{2}$ and $n$ even.

We claim that the four properties enumerated in Theorem 3.4 are also sufficient for the pair $H \supseteq K$ to be the spatiotemporal and spatial symmetries of a hyperbolic periodic orbit for a equivariant oscillators. In fact, we will prove more by showing that the corresponding closed curve may be chosen as a asymptotically stable periodic orbit of a $\Gamma$-equivariant vector field on $\mathbb{T}^{n}$. In order to prove our claim here, we:

(i) Show that if the subgroups $H \supseteq K$ satisfy (a)-(c), there exists a closed curve $\alpha$ in $\operatorname{Fix}(K)$ that is invariant under the action of $H$.

(ii) Construct a $\Gamma$-equivariant vector field $F: \mathbb{T}^{n} \rightarrow \mathbb{R}^{n}$ for which $\alpha$ is an asymptotically stable periodic orbit.

We address these points in Lemmas 3.5 and 3.6.

Lemma 3.5. Let $\Gamma$ be a finite group acting on $\mathbb{T}^{n}$ by permutations. Suppose that $H \supseteq K$ are subgroups of $\Gamma$ that satisfy Theorem 3.4 (a)-(c). Then, there exists a smooth simple closed curve $\alpha$ contained in $\operatorname{Fix}(K)$ with spatial symmetries $K$ and invariant under $H$.

Proof. Let $k=\operatorname{dim} \operatorname{Fix}(K)$. If $k=1$, then $K=H=\Gamma, \operatorname{Fix}(K)=\left\{(\theta, \ldots, \theta) \in \mathbb{T}^{n}\right\}$, and we can choose $\alpha(t)=(t, \ldots, t)$.

If $k=2$ assumption (c) implies (after renumbering of coordinates) that

$$
\operatorname{Fix}(K)=\left\{\left(\theta_{1}, \ldots, \theta_{1}, \theta_{2}, \ldots, \theta_{2}\right) \in \mathbb{T}^{n}\right\},
$$

and either $H=K$ or $H / K=\mathbf{Z}_{2}$ with $H=N_{\Gamma}(K)$. In either case $L_{K}=\left\{(\theta, \ldots, \theta) \in \mathbb{T}^{n}\right\}$ and $C=\operatorname{Fix}(K) \backslash L_{K}$ is connected. In the first case we can choose $\alpha$ to be any closed curve in $C$. In the second case $H$ acts as the permutation symmetry $\left(\begin{array}{ll}1 & 2\end{array}\right)$ on $\operatorname{Fix}(K)=\mathbb{T}^{2}$. We can write $\mathbb{T}^{2}=[0,1] \times[0,1]$ modulo spatial periodicity and choose $\alpha(t)=(t, t+0.5)$. Note that

$$
(12) \alpha(t)=(t+0.5, t) \equiv(s, s+0.5)=\alpha(s)=\alpha(t+0.5)
$$


where $s=t+0.5$. So $\alpha$ is fixed by $K$ and invariant under $H$ as desired.

Finally, assume $k \geq 3$. Let $C$ be a connected component of $\operatorname{Fix}(K) \backslash L_{K}$ that is fixed by $H$, the existence of which is guaranteed by assumption (d). We show that there exists a smooth simple closed curve $\alpha$ such that

(i) $\alpha(t) \in C$ for all $t$,

(ii) $\{\alpha(t)\}$ is $H$-invariant,

(iii) for all $\gamma \in \Gamma \backslash H, \gamma\{\alpha(t)\} \cap\{\alpha(t)\}=\emptyset$.

Indeed, if a simple closed curve $\alpha$ satisfies (i)-(iii), then $H$ is the subgroup of $\Gamma$ that leaves $\alpha$ invariant and $K$ is the subgroup that fixes every point of $\alpha$. First note that $K$ fixes $\alpha$ pointwise by (i) and $H$ fixes $\alpha$ setwise by (ii). Suppose that that $\gamma \alpha\left(t_{0}\right)=\alpha\left(s_{0}\right)$ for some, then $\gamma \in H$ by (iii) so $H$ is the largest subgroup of $\Gamma$ that leaves $\alpha$ invariant. Second, $K$ is the isotropy subgroup of any point in $\operatorname{Fix}(K) \backslash L_{K}$. So, $\gamma \alpha\left(t_{0}\right)=\alpha\left(t_{0}\right)$, implies that $\gamma \in K$ because $\alpha\left(t_{0}\right) \in C$.

We now construct a simple closed curve $\alpha$ that satisfies (i) and (ii). Note that if $H=K$, then any simple closed curve in $C$ will do. So, let $m=|H / K|>1$. Let $h \in H$ be such that $h K$ is a generator of $H / K$. Fix a point $x_{0} \in C$ and a nonzero tangent vector $v_{0} \in T_{x_{0}} \operatorname{Fix}(K)$. Since $C$ is path connected and $h x_{0} \in C$, we may choose a $\mathcal{C}^{\infty}$ smooth curve $\tilde{\alpha}:[0,1] \rightarrow C$ satisfying

$$
\begin{aligned}
\tilde{\alpha}(t) & =(1+t) v_{0} & & t \in[0, \varepsilon] \\
\tilde{\alpha}(t) & =t h v_{0} & & t \in[1-\varepsilon, 1]
\end{aligned}
$$

where $v_{0} \neq 0$. Next, extend $\tilde{\alpha}$ to be a $\mathcal{C}^{\infty}$ smooth curve defined on $[0,1]$. Note that $h \tilde{\alpha}(0)=\tilde{\alpha}(1)$ so that we can define the $\mathcal{C}^{\infty}$ smooth closed curve $\alpha:[0, m] \rightarrow C$ by

$$
\alpha(t)=h^{i} \tilde{\alpha}(t-i) \quad \text { for } i \leq t \leq i+1 \text { and } 0 \leq i \leq m-1 .
$$

Clearly $\alpha$ is a closed curve inside $C$; while properties (i) and (ii) hold by construction. However, there are two obstructions to $\alpha$ being a simple closed curve: $\alpha$ may have self-intersections and $\gamma \alpha$ may intersect $\alpha$ for some $\gamma \in \Gamma \backslash H$. Since we are assuming that $\operatorname{dim} \operatorname{Fix}(K) \geq 3$ and the groups are finite, we can use transversality arguments to circumvent these difficulties and choose $\tilde{\alpha}$ such that the resulting curve $\alpha$ has no self-intersections and $\gamma \alpha$ does not intersect $\alpha$ for all $\gamma \in \Gamma \backslash H$.

Suppose that $\alpha$ has a self-intersection; that is, $\alpha(r)=\alpha(s)$ for some $r \neq s$. Then, the map

$$
A(t, u)=(\alpha(t), \alpha(u))
$$

intersects the diagonal $\triangle=\{(x, x): x \in \operatorname{Fix}(K)$ at $(r, s)$. Since the domain of $A$ is two-dimensional and the codimension of $\triangle$ is $k>2$, it follows that $A \bar{\Pi} \triangle$ implies $\alpha$ has no self-intersections. We cannot apply the transversality theorem directly because, by construction, $\alpha$ is not arbitrary. Note that, again by construction, $\alpha(r)=\alpha(s)$ if

$$
h^{i} \tilde{\alpha}(r-i)=h^{j} \tilde{\alpha}(s-j)
$$

and hence that

$$
\tilde{\alpha}(\tilde{r})=h^{m} \tilde{\alpha}(\tilde{s})
$$

for some $m$ and $\tilde{r}, \tilde{s} \in[0,1]$. Thus, $\alpha$ has a self inspection only if one of the maps

$$
\tilde{A}_{m}(\tilde{t}, \tilde{u})=\left(\tilde{\alpha}(\tilde{t}), h^{m} \tilde{\alpha}(\tilde{u})\right)
$$

intersects the diagonal $\triangle$. Moreover, $\tilde{\alpha}$ is arbitrary away from the end points and a countable intersection of transversality conditions on $\tilde{\alpha}$ can always be satisfied. So there exists $\tilde{\alpha}$ that leads to a non-self-intersecting $\alpha$, as desired. 
Finally, we can verify (iii) by using another transversality argument. Suppose that $\alpha(r)=\gamma \alpha(s)$ for some $r \neq s$ and $\gamma \in \Gamma \backslash H$. It follows that (iii) is in valid if and only if the map

$$
(\tilde{t}, \tilde{u}) \mapsto\left(\tilde{\alpha}(\tilde{t}), h^{-i} \gamma h^{j} \tilde{\alpha}(\tilde{u})\right)
$$

intersects the diagonal $\triangle$. Thus (iii) is verified by applying the transversality theorem simultaneously for each such map.

We now prove an extension lemma that when combined with the existence lemma above, leads to a proof of the converse of Theorem 3.4.

Lemma 3.6. Let $K \subset \Gamma$ be an isotropy subgroup of the action of $\Gamma$ on $\mathbb{T}^{n}$. Let $\alpha$ be a simple closed curve in $\operatorname{Fix}(K) \backslash L_{K}$ and let $H \subset N_{\Gamma}(K)$ be the subgroup of $\Gamma$ that preserves $\alpha$ setwise. Then, there exists a $\Gamma$-equivariant vector field $F$ on $\mathbb{T}^{n}$ such that $\alpha$ is an asymptotically stable periodic orbit for $F$.

Proof. Choose an $H$-invariant tubular neighborhood $U$ of $\alpha$ such that $\gamma U \cap U=\emptyset$ for all $\gamma \notin H$. Near $\alpha$ we can write $U \cong \mathbb{T} \times \mathbb{R}^{n-1}$ where $h(\theta, v)=(h \theta, h v)$ for all $h \in H$.

Let $G_{1}$ be a nonzero vector field defined on $\alpha$ that is tangent to $\alpha$. Let

$$
G_{2}(\theta)=\frac{1}{|H|} \sum_{h \in H} h^{-1} G_{1}(h \theta)
$$

where $\theta$ is on $\alpha$. Then $G_{2}$ is a nonzero $H$-equivariant vector field defined on $\alpha$ that is tangent to $\alpha$. Next, extend $G_{2}$ to a vector field $G_{3}$ defined on $U$ so that $\alpha$ is an asymptotically stable limit cycle for $G_{2}$ and $G_{2}=0$ near the boundary of $U$. Indeed we can take $G_{3}(\theta, v)=(\alpha(\theta),-v)$ on $\alpha$. We can then extend $G_{3}$ to be a smooth vector field $G_{4}$ on $\mathbb{T}^{n}$ that is zero off $U$. Finally, average $G_{4}$ over $\Gamma$ to obtain

$$
F(x)=\frac{1}{|H|} \sum_{\gamma \in \Gamma} \gamma^{-1} G_{4}(\gamma x)
$$

Observe that $F$ is $\Gamma$-equivariant and on the tubular neighborhood $U$

$$
F(u)=\frac{1}{|H|} \sum_{\gamma \in \Gamma} \gamma^{-1} G_{4}(\gamma u)=\frac{1}{|H|} \sum_{\gamma \in H} \gamma^{-1} G_{3}(\gamma u)
$$

since $G_{4}(\gamma u)=0$ if $\gamma \notin H$. Finally, near $\alpha$

$$
F(\theta, v)=\frac{1}{|H|} \sum_{\gamma \in H} \gamma^{-1}\left(\gamma G_{2}(\theta),-\gamma v\right)=\frac{1}{|H|} \sum_{\gamma \in H}\left(G_{2}(\theta),-v\right)=\left(G_{2}(\theta),-v\right)
$$

since $G_{2}$ is $H$-equivariant. Hence, $\alpha$ is an asymptotically stable limit cycle for $F$, as desired.

Proof of sufficiency for Theorem 3.4: We now prove that the necessary conditions enumerated in Theorem 3.4 for a pair of subgroups $H \supseteq K$ to be the symmetries of a periodic solution on $\mathbb{T}^{n}$ are also sufficient for the existence of a periodic solution on $\mathbb{T}^{n}$ having $H \supseteq K$ as it symmetries pair. More precisely, we show that such a curve may be chosen as a locally attracting periodic solution of a smooth $\Gamma$-equivariant vector field. Lemma 3.6 implies that there exists a $\Gamma$-equivariant vector field $F$ that has $\alpha$ as an asymptotically stable limit cycle, as desired. 


\section{Comparing menus of admissible pairs $H \supseteq K$}

We now have all the ingredients for comparing the menus of admissible pairs supported by equivariant equations, equivariant oscillators, and equivariant systems.

Theorem 3.7. Let $\Gamma$ be the symmetry group of a coupled-cell network. Let $H \supseteq K$ be subgroups of $\Gamma$. Then:

(a) If the pair $H \supseteq K$ are the symmetries of a periodic solution of some $\Gamma$-equivariant equation, then some $\Gamma$-equivariant oscillator has a periodic solution with spatiotemporal symmetries $H$ and spatial symmetries $K$.

(b) If the pair $H \supseteq K$ are the symmetries of a periodic solution of some $\Gamma$-equivariant oscillator, then some $\Gamma$-equivariant system has a periodic solution with spatiotemporal symmetries $H$ and spatial symmetries $K$.

Proof. The comparisons are done by checking the necessary and sufficient conditions in the $H / K$ theorem for each class. Theorem 3.4 implies that to verify (a) we need to check that $H$ fixes a connected component of $\operatorname{Fix}_{\mathbb{T}}(K) \backslash L_{K}^{\mathbb{T}}$. Let $\pi: \mathbb{R}^{k} \rightarrow \mathbb{T}^{k}$ be the quotient map defining the topology of $\mathbb{T}^{k}$ and $C_{\mathbb{R}}$ a connected component of $\operatorname{Fix}_{\mathbb{R}}(K) \backslash L_{K}^{\mathbb{R}}$ fixed by $H$; the existence of which is guaranteed by Theorem 3.3. Note that

$$
\pi\left(\operatorname{Fix}_{\mathbb{R}}(K) \backslash L_{K}^{\mathbb{R}}\right)=\operatorname{Fix}_{\mathbb{T}}(K) \backslash L_{K}^{\mathbb{T}} .
$$

We claim that the connected component of $\operatorname{Fix}_{\mathbb{T}}(K) \backslash L_{K}^{\mathbb{T}}$ containing $\pi\left(C_{\mathbb{R}}\right)$ is fixed by $N_{\Gamma}(K)$. Indeed, since the actions of $N_{\Gamma}(K)$ on $\operatorname{Fix}_{\mathbb{R}}(K)$ and $\operatorname{Fix}_{\mathbb{T}}(K)$ commute with $\pi$, that is, $\left.\pi\right|_{\operatorname{Fix}_{\mathbb{R}}(K)}$ is $N_{\Gamma}(K)$-equivariant, it follows that $\pi\left(C_{\mathbb{R}}\right)$ is fixed by $H$. But each element $h \in H$ permutes the connected component of $\operatorname{Fix}_{\mathbb{T}}(K) \backslash L_{K}^{\mathbb{T}}$, hence the connected component containing $\pi\left(C_{\mathbb{R}}\right)$ is fixed by $H$.

To prove assertion (b), it suffices to note that the necessary and sufficient conditions for pairs of symmetries of periodic solutions on equivariant oscillators are a subset of those needed for the same pair to be the symmetries of some periodic orbit on equivariant systems.

Example 3.8. We claim that in general the containments listed in the top row of Figure 3 are strict. Consider the all-to-all coupled three identical node network with symmetry group $\Gamma=\mathbb{S}_{3}$.

(a) The pair $(H, K)=\left(\mathbb{S}_{3}, \mathbb{S}_{3}\right)$ is a solution type that occurs for equivariant coupled oscillators but does not occur in equivariant coupled equations. Note that $\Gamma$-equivariant vector fields on $\mathbb{R}^{3}$ cannot support this symmetry pair since $\operatorname{Fix}(K) \cong \mathbb{R}$. On the other hand, Theorem 3.4 implies that such hyperbolic solutions are supported on $\mathbb{T}^{3}$ since $\operatorname{Fix}(K) \cong \mathbb{T}^{1}$.

(b) Similarly, we claim that the pair $(H, K)=\left(\mathbf{Z}_{2}(12), \mathbf{1}\right)$ is a solution type that occurs for equivariant coupled systems but does not occur for equivariant coupled oscillators. For equivariant systems there are no obstructions on a symmetry pair beyond the algebraic conditions $K=\mathbf{1}$ is an isotropy subgroup and $H / K \cong \mathbf{Z}_{2}\left(\begin{array}{ll}1 & 2\end{array}\right)$ is cyclic, which are both valid. Therefore, Corollary 3.2 implies that this pair is supported on $\mathbb{S}_{3}$-equivariant vector fields on $\left(\mathbb{R}^{k}\right)^{3}$ where $k \geq 2$. We claim that no such vector field may be found on $\mathbb{T}^{3}$. First we note that regardless of the ambient space, the variety $L_{\mathbf{1}}$ is given by

$$
L_{\mathbf{1}}=\left\{y_{1}=y_{2}\right\} \cup\left\{y_{2}=y_{3}\right\} \cup\left\{y_{1}=y_{3}\right\} .
$$


It is easy to check that $L_{\mathbf{1}}$ divides the 3 -torus $\operatorname{Fix}(\mathbf{1})=\mathbb{T}^{3}$ into two connected components as follows:

$$
C_{1}: y_{1}<y_{2}<y_{3} \quad \text { or } \quad y_{2}<y_{3}<y_{1} \quad \text { or } \quad y_{3}<y_{1}<y_{2}
$$

and

$$
C_{2}: y_{1}<y_{3}<y_{2} \quad \text { or } \quad y_{2}<y_{1}<y_{3} \quad \text { or } \quad y_{3}<y_{2}<y_{1}
$$

where $0 \leq y_{1}, y_{2}, y_{3}<1$. Moreover, these components are swapped by the transposition (1 2) in $H$. Therefore, Theorem 3.4 (c) fails and there is no $\mathbb{S}_{3}$-equivariant vector field on $\mathbb{T}^{3}$ supporting a periodic solution with spatiotemporal symmetries $H=\mathbf{Z}_{2}(1,2)$ and trivial spatial symmetry.

\section{Symmetry pairs for admissible vector fields on networks}

We fix a coupled-cell network $\mathcal{G}$ with nontrivial symmetry group $\Gamma$. It is a fact that every admissible vector field is $\Gamma$-equivariant. However, in general, there are two ways in which admissible vector fields differ from equivariant vector fields.

First, networks are not in general all-to-all coupled. In these cases the coordinates of admissible vector fields depend only on a subset of coordinates. However, the corresponding equivariant vector fields are all-to-all coupled in the sense that each vector field coordinate can depend on all other coordinates.

Second, admissible vector field node coordinates may satisfy more invariance conditions than $\Gamma$-equivariant vector fields. For example, consider the five-node ring in Figure 4. Let

$$
\dot{x}_{1}=f_{1}\left(x_{1}, x_{2}, x_{3}, x_{4}, x_{5}\right)
$$

be the first coordinate of a vector field on the phase space of the network. If the vector field is admissible, then $f_{1}$ satisfies the two invariance conditions

$$
f_{1}\left(x_{1} ; x_{2}, x_{3}, x_{4}, x_{5}\right)=f_{1}\left(x_{1} ; x_{2}, x_{4}, x_{3}, x_{5}\right) \text { and } f_{1}\left(x_{1} ; x_{2}, x_{3}, x_{4}, x_{5}\right)=f_{1}\left(x_{1} ; x_{5}, x_{3}, x_{4}, x_{2}\right)
$$

whereas the first coordinate of a $\mathbf{D}_{5}$-equivariant vector field satisfies only the single condition

$$
f_{1}\left(x_{1} ; x_{2}, x_{3}, x_{4}, x_{5}\right)=f_{1}\left(x_{1} ; x_{5}, x_{4}, x_{3}, x_{2}\right)
$$

Thus, equivariant vector fields lead to those $f_{1}$ that are invariant under only the single permutation $(25)(34)$, whereas admissible vector fields lead to those $f_{1}$ that are invariant under both transpositions (25) and (34). Clearly, admissible vector fields are equivariant, but not conversely.

\section{When are subgroup pairs $H \supset K$ admissible for admissible systems?}

Fix a pair of subgroups $H \supset K$ that are the symmetry groups of a periodic solution of a $\Gamma$ equivariant vector field. We now ask whether $H \supset K$ can be the symmetry groups of some admissible vector field. This question is valid in each of the three kinds of networks we consider: coupled equations, coupled oscillators, and coupled systems. Note that the converse is always valid since admissibles are $\Gamma$-equivariant. 


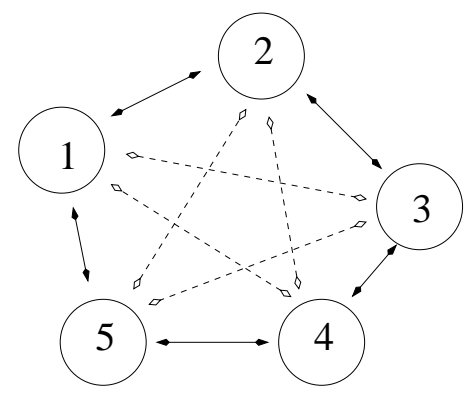

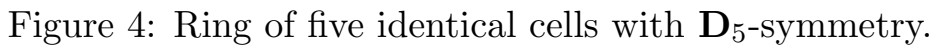

\section{The Josić-Török result for admissible coupled systems}

Recall that a network is transitive (or path-connected or strongly connected) if every pair of nodes is connected by a path of arrows. Josić and Török [17] proved that for coupled systems the equivariant classification extends to the admissible one. Specifically:

Theorem 4.1 ([17, Theorem 18]). Let $\Gamma$ be the symmetry group of a transitive coupled-cell network $\mathcal{G}$. The pair of subgroups $H \supseteq K$ of $\Gamma$ are the symmetries of a hyperbolic periodic solution of an admissible coupled system on $\mathcal{G}$ if and only if

a) $H / K$ is cyclic and

b) $K$ is an isotropy subgroup of $\Gamma$.

Furthermore, the admissible coupled system can be chosen so that the periodic solution is asymptotically stable inside $\operatorname{Fix}(K)$.

In Example 4.2 we show that the analogous theorem is false for coupled equations on transitive networks. We have not determined whether admissible pairs for equivariant oscillators and coupled oscillators are different or the same.

Example 4.2 (Equivariant and admissible classifications differ for coupled equations). Consider the transitive four-cell network in Figure 1(b) with $\Gamma=\mathbf{Z}_{2}(\sigma)$ symmetry, where $\sigma=\left(\begin{array}{lll}1 & 2\end{array}\right)\left(\begin{array}{ll}3 & 4\end{array}\right)$. We set $H=\mathbf{Z}_{2}(\sigma)$ and $K=\mathbf{1}$ and show that there exists a $\Gamma$-equivariant system with a periodic solution having $H, K$ symmetries, but there does not exist such an admissible system. This example shows that $\subsetneq$ in the first column of Figure 3 is correct.

The equivariance result follows from the $H / K$ Theorem. Specifically, $H / K$ is cyclic; $K$ is an isotropy subgroup; $\operatorname{dim} \operatorname{Fix}(K)=4>2$; and $L_{K}=\left\{x \in \mathbb{R}^{4}: x_{1}=x_{2}, x_{3}=x_{4}\right\}$ so that $\operatorname{Fix}(K) \backslash L_{K}=\mathbb{R}^{4} \backslash L_{K}$ is connected. Now apply Theorem 3.1.

Next consider admissible coupled equations that have the form (1.2). We claim that there cannot exist a $T$-periodic solution of (1.2) that satisfies

$$
\begin{aligned}
& x_{2}(t)=x_{1}\left(t+\frac{1}{2} T\right) \\
& x_{4}(t)=x_{3}\left(t+\frac{1}{2} T\right) .
\end{aligned}
$$

To verify the claim, observe that

$$
\triangle=\left\{\left(x_{1}, x_{2}, x_{3}, x_{4}\right): x_{1}=x_{2}\right\}
$$

is a synchrony subspace. Suppose that there exists a $T$-periodic solution to (1.2) satisfying (4.1). Because the $x_{1} \in \mathbb{R}$ it follows that there is a time $t_{0}$ such that

$$
x_{1}\left(t_{0}\right)=x_{1}\left(t_{0}+\frac{1}{2} T\right) .
$$


It then follows from (4.1) that $x_{2}\left(t_{0}\right)=x_{1}\left(t_{0}\right)$. Flow invariance of $\triangle$ then implies that $x_{2}(t)=x_{1}(t)$ for all $t$; that is, the periodic solution $x(t)$ lies in $\triangle$.

The quotient network corresponding to $\triangle$ is given in Figure 2 and has a symmetry $\tau=(34)$. The $H / K$ Theorem for coupled equations guarantees that admissible equations for this network cannot have periodic solutions satisfying $x_{4}(t)=x_{3}\left(t+\frac{1}{2} T\right)$ since on the quotient network $L_{1}=$ $\left\{\left(x_{1}, x_{3}, x_{4}\right): x_{3}=x_{4}\right\}$ has codimension one and $\tau$ interchanges the two connected components of $\mathbb{R}^{3} \backslash L_{1}$. Hence solutions satisfying (4.1) do not exist for coupled equations even though they do exist for $\mathbf{Z}_{2}(\sigma)$-equivariant systems.

We note that this network is not a counterexample for Theorem 4.1 in the case of coupled oscillators since $\mathbb{T}^{3} \backslash L_{1}$ is connected and appropriate admissible vector fields on the quotient network do exist.

\section{Comparison of the $H \supset K$ menus of admissible pairs}

Although we have not characterized the admissible pairs for admissible vector fields, we are able to prove that the menus of admissible pairs in the three cases of coupled equations, coupled oscillators, and coupled systems enjoy the same hierarchy as for equivariant vector fields.

Theorem 4.3. Let $\mathcal{G}$ be a coupled-cell network with symmetry group $\Gamma$. Suppose that a pair of subgroups $H \supseteq K$ of $\Gamma$ is admissible for coupled equations. Then it is also admissible for coupled oscillators.

Proof. Suppose that the admissible system of coupled equations

$$
\dot{x}_{i}=f_{i}\left(x_{i}, x_{I(i)}\right) \text {, }
$$

where $x_{I(i)}$ is the set of input variables to cell $i$, possesses a periodic orbit $x(t)$ with symmetry groups $H$ and $K$. After a suitable translation $F(X-\alpha)$, if necessary, we may assume that the coordinates functions of $x(t)$ are all positive. We construct an admissible system of coupled oscillators $G=$ $\left(g_{1}, \ldots, g_{n}\right)$ from $F=\left(f_{1}, \ldots, f_{n}\right)$ having $x(t)$ as a periodic orbit. The periodic orbit $x(t)$ is bounded; therefore we can chose $0<r<\frac{R}{2}$ so that for each $i$

$$
r<x_{i}(t)<R / 2 \text { for all } t
$$

By rescaling the vector field $F(x) \rightarrow F(R x)$, we can assume $R=1$.

Let $\phi: \mathbb{R} \rightarrow[0,1]$ be a bump function supported in $(0,1)$ such that

$$
\phi(s)=1 \quad \text { for } \quad r<s<\frac{1}{2} .
$$

Define the mapping $\tilde{G}$ by

$$
\tilde{G}_{i}\left(x_{1}, x_{I(i)}\right)=\phi\left(x_{i}\right) f_{i}\left(x_{i}, x_{I(i)}\right), \quad 1 \leq i \leq n .
$$

The function $\tilde{G}$ is a smooth vector field on $\mathbb{R}^{n}$ supported away from the boundary in the cube $[0,1]^{n}$. As such, $\tilde{G}$ defined on the cube is easily extended to a periodic vector field $G$ on $\mathbb{R}^{n}$.

It is easy to verify that $G$ is admissible since the localizing factor $\phi$ only depends on the internal state variable. Also, $x(t)$ is a periodic orbit of $G$ since $\{x(t)\} \subset(r, 1 / 2)^{n}$ and $\phi[r, 1 / 2]=1$. Finally, since $(r, 1 / 2)^{n}$ is open in $\mathbb{T}^{n} \cong \mathbb{R}^{n} / \mathbf{Z}^{n}$, we infer that $\{x(t)\}$ is a solution of the admissible vector field $G$ of coupled oscillators. 
Next, we show that for a given coupled-cell network, the admissible pairs $H \supset K$ for coupled oscillators are also admissible for coupled systems.

Theorem 4.4. Let $\mathcal{G}$ be a coupled-cell network with symmetry group $\Gamma$. Suppose that a pair of subgroups $H \supseteq K$ of $\Gamma$ is admissible for coupled oscillators. Then the pair is also admissible for coupled systems.

Proof. Suppose that the pair of subgroups $H \supseteq K$ of $\Gamma$ is admissible for coupled oscillators on a coupled-cell network $\mathcal{G}$. Let $G$ be an admissible vector field on $\mathbb{T}^{n}$ possessing a hyperbolic periodic orbit $\Theta(t)=\left(\theta_{1}(t), \ldots, \theta_{n}(t)\right)$ with symmetry groups $H$ and $K$. Denote the coordinate functions of $G$ by

$$
\dot{\theta}_{i}=g_{i}\left(\theta_{i}, \theta_{I(i)}\right)
$$

where $1 \leq i \leq n$.

Let

$$
\hat{M}=\mathbb{R}^{k_{1}} \times \cdots \times \mathbb{R}^{k_{n}},
$$

where $k_{i} \geq 2$, be the phase space for the coupled systems. It suffices to construct the desired coupled systems vector field $F$ on $M=\left(\mathbb{R}^{2}\right)^{n}$, because then we can extend $F$ to $\hat{F}$ on $\hat{M}$ by writing $\mathbb{R}^{k_{1}}=\mathbb{R}^{2} \times \mathbb{R}^{k_{i}-2}$ and setting $\hat{F}_{i}(x, y)=\left(F_{i}(x),-y_{i}\right)$. Note that $M$ is globally attracting as a submanifold of $\hat{M}$ and it suffices to construct $F$ on $M$ from $G$ on $\mathbb{T}^{n}$.

Next, note that

$$
\mathbb{T}^{n}=\left\{\left(x_{1}, \ldots, x_{n}\right) \in\left(\mathbb{R}^{2}\right)^{n}:\left|x_{1}\right|=\cdots=\left|x_{n}\right|=1\right\}
$$

is an embedded submanifold of $\left(\mathbb{R}^{2}\right)^{n}$. We extend $G$ to an admissible vector field $F$ on $\left(\mathbb{R}^{2}\right)^{n}$ for which

$$
X(t)=\left(x_{1}(t), \ldots, x_{n}(t)\right) \text { with } x_{i}(t)=\left(\cos \theta_{i}(t), \sin \theta_{i}(t)\right)
$$

is a hyperbolic periodic orbit. Note that $X(t)$ has $K$ as its spatial symmetries group and $H$ as its invariant group. Let $f: \mathbb{R} \rightarrow \mathbb{R}$ satisfy $f(1)=0$ and $f^{\prime}(1)=-1$. Using polar coordinates on each nodal phase space $\mathbb{R}^{2}$, we define the vector field $F$ on $\left(\mathbb{R}^{2}\right)^{n}$ as follows

$$
\begin{aligned}
\dot{r}_{i} & =f\left(r_{i}\right) \\
\dot{\theta}_{i} & =g_{i}\left(\theta_{i}, \theta_{I(i)}\right)
\end{aligned} \quad 1 \leq i \leq n
$$

The vector field $F$ is admissible since the $\dot{r}_{i}$ coordinates are identical for each node and $G$ is admissible in the $\theta_{i}$ coordinates. Furthermore, the torus $\mathbb{T}^{n}$ is attracting for $F$ and the curve $X(t)$ defined in (4.2) is a periodic solution of $F$ inside $\mathbb{T}^{n}$. Finally, $X(t)$ is a hyperbolic periodic orbit for $F$ since $\Theta(t)$ is hyperbolic for $G, F$ extends $G$ to $\left(\mathbb{R}^{2}\right)^{n}$, and $\mathbb{T}^{n}$ is globally attracting for $F$. Thus, $H \supseteq K$ is an admissible symmetry pair for coupled systems.

We have now verified all of the containments in Figure 3.

\section{Phase-shift synchrony}

As discussed in the introduction, the symmetries in $K$ correspond to synchrony and the symmetries in $H$ correspond to phase-shift synchrony and multirhythms. The results of $[20,21,8,9]$ show that in the cases of coupled equations and coupled systems all patterns of phase-shift synchrony arise from a symmetry on some quotient network. The corresponding statement has not been proved in the case of coupled oscillators. If the statement for coupled oscillators were valid, then we could 
easily compare patterns of phase-shift synchrony in the three contexts using $H / K$ theory. However, using a different argument we can show that patterns for coupled equations are patterns for coupled oscillators and systems (Theorem 5.1).

\section{Comparison of patterns for equations with patterns for oscillators and systems}

Assuming the network is transitive, patterns of rigid phase-shift synchrony for coupled equations and coupled oscillators are each forced by admissible cyclic symmetries on quotient networks. This follows from the results in the series of papers [20, 21, 8, 9] mentioned previously. The result for coupled systems is an "if and only if" result in the sense that there is a coupled system admissible vector field with a periodic solution that exhibits any given pattern of synchrony implied by a symmetry on the quotient network. ${ }^{1}$ As we showed (see Example 4.2) periodic solutions corresponding to cyclic symmetries on quotient networks need not exist for coupled equations since in this context they can be obstructed by the $H / K$ Theorem (Corollary 3.3) applied to the quotient network. What we can show is that each pattern of synchrony that is realizable for coupled equations is realizable for both coupled oscillators and coupled systems.

Theorem 5.1. Let $\mathcal{G}$ be a coupled cell network and let $F$ be an admissible vector field for coupled equations with a periodic solution that has a pattern of phase-shift triple. Then there exist admissible vector fields $G$ for coupled oscillators and for coupled systems that has the same pattern of phaseshift synchrony.

Proof. The result for coupled oscillators is a corollary of the proof of Theorem 4.3. In that proof we showed that if $F$ has a periodic solution $X(t)$ with certain phase-shifts, it can be embedded in the torus (because the periodic solution is bounded and can be assumed to live in the unit cube) and be the solution of a vector field $G$ on the $n$-torus. In fact, more is true. The embedding of $F$ to $G$ is $1: 1$ for perturbations of $F$ on a neighborhood of the trajectory $\{X(t)\}$. Hence, rigidity on the coupled equations level leads to rigidity on the coupled oscillators level, and the theorem is proved.

The result for coupled systems follows from the fact that in both contexts the relevant patterns of phase-shift synchrony are generated by cyclic symmetries on quotient networks and the fact mentioned above that all such symmetries generate patterns of phase-shift synchrony for coupled systems.

Remark 5.2. We believe that each pattern of phase-shift synchrony in coupled oscillators is forced by a symmetry on a quotient network, but this has not yet been shown. This statement is valid if and only if every pattern of phase-shift synchrony that is realizable in coupled oscillators is also realized in coupled systems.

It is the case that there are patterns of phase-shift synchrony for coupled oscillators that are not realizable for coupled equations and patterns for coupled systems that are not realizable for coupled oscillators. This follows directly from the corresponding $H / K$ results (Theorem 3.4 and Corollary 3.3).

\section{Acknowledgments}

The authors thank Ian Stewart and Andrea Barreiro for helpful discussions. This research was supported in part by NSF Grant DMS-0931642 to the Mathematical Biosciences Institute.

\footnotetext{
${ }^{1}$ This result follows from Josić-Török (Theorem 4.1) with the proviso that the constructed periodic solution is hyperbolic on the quotient network, but not necessarily on the full phase space.
} 


\section{References}

[1] Butera Jr, Robert J., John Rinzel, and Jeffrey C. Smith. Models of respiratory rhythm generation in the pre-Bötzinger complex. I. Bursting pacemaker neurons. J. Neurophysiology $\mathbf{8 2 . 1}$ (1999) 382-397.

[2] Butera Jr, Robert J., John Rinzel, and Jeffrey C. Smith. Models of respiratory rhythm generation in the pre-Bötzinger complex. II. Populations of coupled pacemaker neurons. J. Neurophysiology 82.1 (1999) 398-415.

[3] P.-L. Buono and M. Golubitsky. Models of central pattern generators for quadruped locomotion. I. Primary gaits. J. Math. Biol. 42(4) (2001) 291-326.

[4] A.H. Cohen, G.B Ermentrout, T. Kiemel, N. Kopell, K.A. Sigvardt, and T.L. Williams. Modelling of intersegmental coordination in the lamprey central pattern generator for locomotion. Trends in Neurosciences 15 (11) (1992) 434-438.

[5] C.O. Diekman and M. Golubitsky. Network Symmetry and Binocular Rivalry Experiments. $J$. Math. Neuro. 4 (12) (2014) DOI 10.1186/2190-8567-4-12

[6] J. Duysens and H.W.A.A. Van de Crommert. Neural control of locomotion; Part 1: The central pattern generator from cats to humans." Gait \& Posture 7.2 (1998) 131-141.

[7] M. Golubitsky, K. Josić, and E. Shea-Brown. Winding numbers and average frequencies in phase oscillator networks. J. Nonlinear Sci. 16(3) (2006) 201-231.

[8] M. Golubitsky, D. Romano, and Y. Wang. Network periodic solutions: full oscillation and rigid synchrony. Nonlinearity 23 (2010) 3227-3243.

[9] M. Golubitsky, D. Romano, and Y. Wang. Network periodic solutions: patterns of phase-shift synchrony. Nonlinearity 25 (2012) 1045-1074.

[10] M. Golubitsky and I. Stewart. The symmetry perspective: From equilibrium to chaos in phase space and physical space. Progress in Mathematics 200. Birkhäuser Verlag, Basel, 2002.

[11] M. Golubitsky and I. Stewart. Nonlinear dynamics of networks: the groupoid formalism. Bull. Amer. Math. Soc. (N.S.) 43(3) 305-364.

[12] M. Golubitsky, I. Stewart, P.L. Buono, and J.J. Collins. A modular network for legged locomotion. Physica D 115 (1998) 56-72.

[13] M. Golubitsky, I. Stewart, P.L. Buono, and J.J. Collins. Symmetry in locomotor central pattern generators and animal gaits. Nature 401 (1999) 693-695.

[14] M. Golubitsky, M. Nicol and I. Stewart. Some curious phenomena in coupled cell systems. $J$. Nonlinear Sci. 14 (2) (2004) 207-236.

[15] M. Golubitsky, I. Stewart, and D.G. Schaeffer. Singularities and Groups in Bifurcation Theory. Vol. II, Applied Mathematical Sciences 69, Springer-Verlag, New York, 1988.

[16] M. Golubitsky, I. Stewart, and A. Török. Patterns of synchrony in coupled cell networks with multiple arrows. SIAM J. Appl. Dyn. Syst. 4(1) (2005) 78-100.

[17] K. Josić and A. Török. Network architecture and spatio-temporally symmetric dynamics. Physica D 224(1-2) (2006) 52-68.

[18] F.K. Skinner, N. Kopell, and E. Marder. Mechanisms for oscillation and frequency control in reciprocally inhibitory model neural networks. J. Comp. Neuroscience 1.1-2 (1994) 69-87.

[19] I. Stewart, M. Golubitsky, and M. Pivato. Symmetry groupoids and patterns of synchrony in coupled cell networks. SIAM J. Appl. Dyn. Sys. 2 (4) (2003) 609-646.

[20] I. Stewart and M. Parker. Periodic dynamics of coupled cell networks I: Rigid patterns of synchrony and phase relations. Dyn. Syst. 22 (2007) 389-450. 
[21] I. Stewart and M. Parker. Periodic dynamics of coupled cell networks II: Cyclic symmetry. Dyn. Syst. 23 (2008) 17-41.

[22] D. Terman, N. Kopell, and A. Bose. Dynamics of two mutually coupled slow inhibitory neurons. Physica D 117 (1) (1998) 241-275.

[23] X-J Wang and J. Rinzel. Alternating and synchronous rhythms in reciprocally inhibitory model neurons. Neural Comp. 4.1 (1992) 84-97.

[24] H.R. Wilson. Requirements for conscious visual processing. In: Cortical Mechanisms of Vision. (M. Jenkins and L. Harris, eds) Cambridge University Press, 2009, 399-417.

[25] C. Zhang, R.D. Guy, B. Mulloney, Q. Zhang, and T.J. Lewis. Neural mechanism of optimal limb coordination in crustacean swimming. PNAS 111 (38) (2014) 13840-13845. 